\title{
Effets du ramassage des fourrages sur l'environnement et sur les activités agro-sylvo-pastorales et commerciales dans les régions sahéliennes au Niger
}

\author{
Abdou DAN GOMMA ${ }^{1 *}$, Salissou ISSA ${ }^{1}$, Tidjani ABASSE ${ }^{1}$, Maxim BANOIN $^{2}$ et \\ Eva SCHLECHT ${ }^{3}$
}

\author{
${ }^{1}$ Institut National de la Recherche Agronomique du Niger (INRAN), BP 429 Niamey, Niger. \\ ${ }^{2}$ Université Abdou Moumouni de Niamey, BP10 960 Niamey, Niger. \\ ${ }^{3}$ University of Kassel and Georg-August University, Mönchebergstraße 19, 34125 Kassel, Allemagne. \\ *Auteur correspondant ; E-mail : abdoudangoma@yahoo.fr; Tel : (00227) 96985171 /(00227)90430838
}

\section{REMERCIEMENTS}

Les auteurs tiennent à remercier vivement le Programme de Productivité Agricole en Afrique de l'Ouest (PPAAO/WAAPP) pour son appui financier pour la réalisation de la présente étude.

\section{RESUME}

$\mathrm{Au}$ Sahel, pour répondre à la demande croissante en produits animaux, les éleveurs utilisent essentiellement les fourrages naturels sur pieds et récoltés. Cette pratique affecte surtout les écosystèmes périurbains. La présente étude vise à évaluer les effets visibles du prélèvement des fourrages sur l'environnement et sur les activités rurales en zones périurbaines. Une enquête de perception des populations sur les effets de la collecte de paille, conjuguée à la mesure de la matière organique, à l'inventaire forestier, au comptage de surfaces dénudées et de ravins sur un hectare en deux répétions, a été réalisée dans les sites de collecte de 12 villages des régions de Maradi et Tillabéry. Les résultats ont montré que l'amélioration de l'alimentation des animaux, la diversification de source de revenus et de matériaux de la confection d'habitats sont les avantages du ramassage de fourrages. La dégradation des terres, la perte de la biodiversité végétale et à l'appauvrissement des sols constituaient les effets néfastes de la collecte des fourrages, qui étaient plus accentués à Tillabéry qu'à Maradi. Le développement et la promotion des cultures fourragères permettent de répondre à la demande croissante en fourrages des marchés urbains et réduire la collecte des fourrages sur les parcours périurbains.

(C) 2019 International Formulae Group. All rights reserved.

Mots clés : Impact, Collecte, Paille, Dégradation, Maradi, Tillabéry.

\section{Effects of forage harvesting on environmental, agricultural, pastoral and trading activities in the sahelian regions of Niger}

\section{ABSTRACT}

In the Sahel, to meet the growing demand for animal products, pastoralists mainly use natural forage on the ground and harvested. This practice mainly affects peri-urban ecosystems. This study aims to evaluate the visible effects of forage harvesting on the environment and on rural activities in peri-urban areas. A population 
perception survey on the effects of straw collection, combined with the measurement of organic matter, the forest inventory, the counting of bare surfaces and ravines on one hectare in two repetitions, was carried out in collection sites of 12 villages in the Maradi and Tillabéry regions. The results showed that the improvement of animal feed, the diversification of source of income and materials of the confection of habitats are the advantages of the gathering of fodder. Land degradation, loss of plant biodiversity and soil depletion were the adverse effects of forage collection, which was more pronounced in Tillabéry than in Maradi. The development and promotion of fodder crops makes it possible to meet the growing demand for fodder in urban markets and reduce forage collection on peri-urban routes.

(C) 2019 International Formulae Group. All rights reserved.

Keywords: Impact, Collect, straw, Degradation, Maradi, Tillabery.

\section{INTRODUCTION}

Au Sahel, l'élevage joue un rôle central dans l'économie des pays avec une contribution au Produit Intérieur Brut (PIB) de 11 à $15 \%$, dans la sécurité alimentaire et dans le revenu des ménages ruraux (Programme Régional d'Appui au Pastoralisme au Sahel (PRAPS), 2015). Le Niger, pays d'élevage par excellence présente un avantage comparatif dans la sous-région ouest africaine. Le cheptel du Niger est estimé en 2016 à 45 millions de têtes toutes espèces confondues soit 16565658 UBT (Direction de Développement Pastoral (DDP), 2017). L'élevage constitue la deuxième source d'exportation après l'uranium (Ministère de l'Elevage (MEL), 2012). Le développement de l'élevage est limité au Sahel en général, par les contraintes (FAO, 2010a). Le pâturage naturel et les résidus de cultures, caractérisées par des faibles valeurs nutritives surtout en saison sèche qui dure 7 à 8 mois dans l'année, constituent les principales ressources fourragères (FAO-CIRAD, 2008); (Abdou et al., 2017). Ces ressources fourragères sont exploitées sur les parcours, collectées, stockées, vendues et distribuées aux animaux au parc dans leur zone de production ou exportées ailleurs (FAO, 2014). L'agriculture minière, le surpâturage et le ramassage des fourrages fragilisent les écosystèmes, surtout ceux périurbains qui sont exposés aux facteurs de dégradation (érosions, activités humaines et aléas climatiques). La dégradation des terres entraine une chute annuelle de près de $3 \%$ de la production agricole, compromettant ainsi la sécurité alimentaire dans la sousrégion sahélienne (Edwige, 2011).
La présente étude vise à déterminer les effets visibles du prélèvement des fourrages sur l'environnement périurbain et sur les activités socio-économiques des populations en zone périurbaine du Niger. Un accent particulier a été mis sur les principaux effets positifs et négatifs de la collecte des fourrages sur l'environnement périurbain ainsi que les impacts du ramassage des fourrages sur les activités socio-économiques (agriculture, élevage, commerce).

\section{MATERIEL ET METHODES \\ Matériel \\ Localisation des sites de l'étude}

L'étude a été conduite en zones agricole et agro-pastorale dans les régions de Maradi et de Tillabéry en général et en zone périurbaine (rayon de $100 \mathrm{~km}$ à partir de la limite administrative des communautés urbaines) de Maradi et de Niamey en particulier. Ces zones ont un climat du type sahélo-soudanien avec deux saisons: une saison des pluies de juin à septembre (4 mois) et une saison sèche d'octobre à mai ( 8 mois) (Soukaradji et al., 2017). Ces zones ont des pluviométries moyennes annuelles de $507 \mathrm{~mm}$ pour Maradi et $530 \mathrm{~mm}$ pour Niamey (Ministère de l'Agriculture et de l'Elevage, 2016).

Les populations de la zone de l'étude, ont comme principales activités l'agriculture, l'élevage, le commerce et l'artisanat. Les villages et les zones de l'étude sont indiqués dans la Figure 1. 


\section{Matériel et méthodes}

Des enquêtes ont été conduites dans les villages périurbains des zones de prélèvement des fourrages commercialisés à Maradi et à Niamey. Elles ont permis de collecter les données sur l'état de surface des sols et la végétation. Dans chaque zone, 6 villages reconnus pour leur production, le ramassage et la vente des fourrages ont été retenus pour les enquêtes qui ont été conduites pendant deux saisons sèches chaudes (mars à juin en 2016 et en 2017). Un choix raisonné des villages a été fait avec l'appui des services techniques et les associations locales des producteurs ruraux au niveau des communes périurbaines de Maradi et de Niamey.

Les principaux critères de choix des villages étaient: i) villages situés en zone périurbaine de Maradi et de Niamey; ii) villages dont la population est reconnue comme productrice, collectrice et vendeuse des fourrages et iii) population ayant comme principales activités l'agriculture, l'élevage et le commerce.

Les sites d'observations étaient les champs de cultures, les jachères et les aires de pâturage ou enclave pastorale situés au-delà d'un (1) kilomètre du village.

Les six villages de la région de Maradi étaient: Djirataoua, Gadambo, Dan Kari (commune de Djirataoua), Guidan Matche Daya (commune de Dan Issa), El Kolta (commune de Guidan Roumji) et Dadin Kowa Abarchi (commune de Sarkin Yamma). Les six villages de la région de Tillabéry étaient : Garbaye Tombo et Gogueze (commune de Dantchiandou), Gatewane et Warou (commune de Simiri), Mondolo Wali Koira (Commune de Tondi Kiwindi) et Kareygorou (commune de Bitinkoji) (Figure 1).

Dans chaque village, l'enquête a été réalisée en focus groupe au cours des assemblées villageoises regroupant les agriculteurs, agro-éleveurs, éleveurs sédentaires et des transhumants. Ainsi au total
12 questionnaires sur les effets du ramassage des fourrages, ont été administrés dans 12 villages périurbains de Maradi et de Tillabéry. Le questionnaire a abordé entre autres, les effets positifs et négatifs du ramassage de fourrages sur le sol, sur la végétation, sur les activités agro-pastorales et commerciales ainsi que les effets conjugués du ramassage et du changement climatique.

Après chaque entretien avec les villageois, une visite des sites de prélèvement des fourrages choisis au hasard, a été faite sur un transect pour effectuer des observations et évaluer les effets du ramassage sur le sol, la végétation et mesurer la biomasse végétale appétible. La superficie d'un hectare, sur laquelle les mesures ont été faites, a été délimitée au moyen d'un Global Positioning System(GPS) de marque GARMIN eTrex 30x. Cinq placettes/ha (1 placette élémentaire $=1 \mathrm{~m}^{2}$ ) avec 2 répétitions par site, ont été posées au milieu de l'hectare et à 50 mètres sur les médianes. La pesée de toute la matière organique végétale de surface sans le bois a été réalisée avec un peson électronique de portée $40 \mathrm{~kg}$ et de $5 \mathrm{~g}$ de précision. Pour l'estimation des ligneux (arbres et arbustes), l'inventaire forestier de nombre de pieds à l'hectare a été faite par comptage manuel.

\section{Analyse statistique}

Les données qualitatives des enquêtes de perception des effets de ramassage des fourrages, ont été saisies avec le logiciel CS Pro 7.0 et l'analyse descriptive a été réalisée avec le logiciel STATA 14. Pour les données quantitatives caractérisant les sites de ramassage des fourrages (unités de surfaces dénudées, ravins, ligneux et matière organique de surface par unité de surface), l'analyse de la variance a été faite selon la modèle General Linear Model (GLM) avec le logiciel SPSS Statistic-19 et la comparaison des différentes moyennes a été faite avec le test de Least Significant Difference (LSD) au seuil de 5\%. 


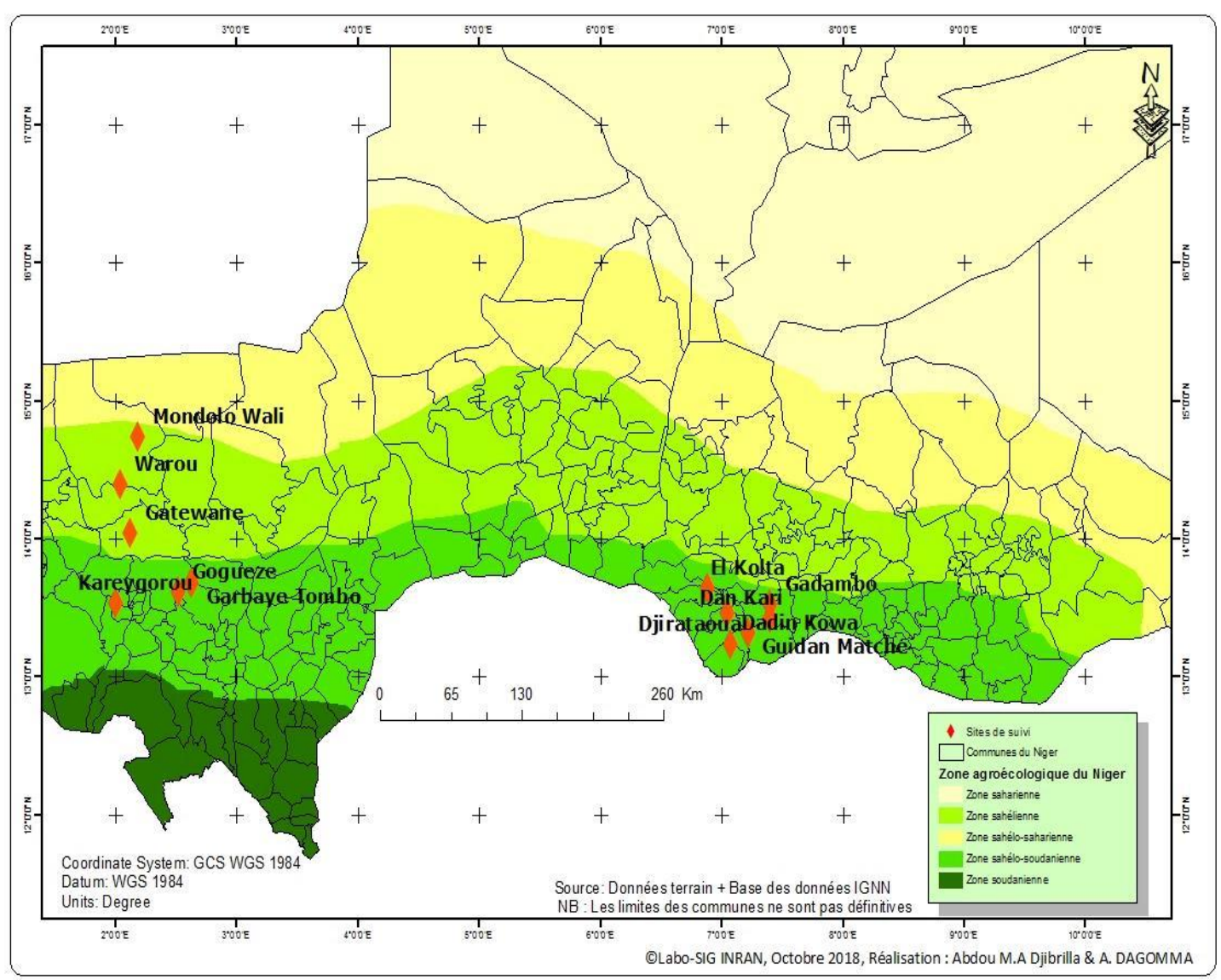

Figure 1 : Localisation des villages de l'étude dans les zones agro-écologiques du Niger.

\section{RESULTATS}

Perception des populations rurales sur les effets du ramassage des fourrages sur l'environnement

La perception des populations sur les effets du ramassage des fourrages (naturels, résidus de cultures et ligneux) a été enregistrée au cours d'un focus groupe en assemblée villageoise dans les 12 villages concernés des régions de Maradi et de Tillabéry.

Les principaux résultats des avantages du ramassage des fourrages sont consignés dans le Tableau 1. Les principaux avantages $\mathrm{du}$ ramassage des fourrages sont ceux qui améliorent la productivité des activités agrosylvo-pastorales et commerciales telles que la génération de revenu monétaire, l'amélioration des productions animales, fonds de financement des autres activités productives (Tableau 1).

Les inconvénients de la collecte des fourrages sur l'environnement et sur les activités humaines sont donnés dans le Tableau 2. Les effets néfastes de la collecte des fourrages réduisent les performances de plusieurs domaines d'activités agro-sylvopastorales et commerciales. Il s'agit de la dégradation des terres, la perte de la biodiversité et la rareté des ressources fourragères sur les parcours (Tableau 2).

\section{Effets du ramassage des fourrages sur la} flore des sites de prélèvement

Le ramassage contribue à la destruction et à l'exportation des semences des espèces fourragères. Certaines espèces sont menacées de disparition alors que d'autres ont disparu par la surexploitation abusive des populations 
et des animaux, et sont été remplacées par les espèces envahissantes peu ou non appétées (Tableau 3).

Il ressort des résultats des enquêtes dans les 12 villages que 27 espèces fourragères sont menacées de disparition dont 12 espèces dans la région de Maradi et 15 dans celle de Tillabéry. L'inventaire avec les populations a fait apparaître la disparition de 12 espèces appétées dont 8 dans la région de Tillabéry et 4 dans la région de Maradi (Tableau 3). Dans les 2 régions, 5 espèces herbacées envahissantes peu ou non appétées ont apparu dans les enclaves pastorales et les jachères, par contre 2 ligneux fourragers (Annona senegalensis et Sclérocaria birrea) ont aussi apparu dans les terroirs de Gatewane, El Kolta et Gadambo.

\section{Effets négatifs conjoints du ramassage des fourrages et du changement climatique}

Sept (7) effets négatifs conjoints du changement climatique et du ramassage des fourrages sont rapportés dans le Tableau 4. La pluviométrie conditionne la production végétale qui est la principale source de fourrages. Les effets négatifs conjoints du changement climatique et du ramassage de la biomasse végétale de surface sont plus ressentis en élevage. Soixante-huit pourcent des effets négatifs portaient sur la sécurité alimentaire et nutritionnelle du bétail et des producteurs tandis que trente-deux pourcent étaient relatifs à la dégradation de l'environnement (Tableau 4).

\section{Effets de ramassage de fourrages sur l'état de la surface du sol des sites:}

Le suivi des transects dans les terroirs villageois ont permis de collecter les données sur la densité des ravins, des unités dénudées de sol par comptage, celle des ligneux par l'inventaire forestier et sur la matière organique végétale de surface dans les champs de cultures, les jachères et les enclaves pastorales par la méthode de récolte intégrale sur 5 placettes d'un mètre carré par hectare par site.

Les résultats de l'analyse statistique ont montré une différence significative entre les sites pour la densité des unités de surface dénudées à l'hectare $(\mathrm{p}=0,004)$ mais la densité des ravins est similaire sur tous les sites $(p=0,264)$. Les aires de pâturage présentaient plus d'indices de dégradation du sol, qui étaient relativement plus importants à Tillabéry. Les champs et les jachères de Maradi ont moins d'unités de surface dénudées que ceux de la région de Tillabéry (Tableau 5).

Effets du ramassage des fourrages sur la quantité de la matière organique à la surface du sol et sur la densité des ligneux dans les sites

Les moyennes de matière organique végétale de surface étaient significativement différentes entre les sites $(\mathrm{p}=0,01)$. La plus grande moyenne a été obtenue dans les champs de cultures suivie de celle des jachères ; les aires de pâturage avaient la plus faible quantité de biomasse de surface à l'hectare. La densité des ligneux était similaire ( $p>0,05)$ dans les champs, les jachères et les aires de pâturage (Tableau 6).

La présence de la matière organique végétale (litière) attenue l'érosion hydrique et éolienne. Certains terroirs éloignés présentent plus de matière organique de surface $(\geq 100 \mathrm{~kg}$ $\mathrm{ha}^{-1}$ ) comme Mondolo, Warou, Gatewane et El Kolta que ceux proches des centres urbains tels que Djirataoua, Guidan Matché Daya, Dan Kari, Dadin Kowa, Gogueze et Kareygorou. Dans la majorité des terroirs, les champs de cultures, suivis des jachères, ont plus de litière que les aires de pâturage qui ont plus de la matière organique végétale dans la région de Tillabéry que les terroirs de Maradi. Dans la zone de Maradi, seuls les sites d'El Kolta ont une quantité de matière organique végétale de surface supérieure ou égale $100 \mathrm{~kg}$ $\mathrm{ha}^{-1}$ alors que ceux de Tillabéry à l'exception de Karegorou et Gogueze, ont des valeurs supérieures (Figure 2). Les faibles quantités de matière organique végétale de surface ont été enregistrées au niveau des terroirs plus proches des centres urbains. Les 2 terroirs de Djirataoua (Maradi) et Kareygorou (Tillabéry) caractérisés par 2 systèmes de production agricole (pluvial, irrigué) avaient une quantité 
de matière organique végétale inférieure ou égale à $80 \mathrm{~kg} \mathrm{ha}^{-1}$ en saison sèche dans tous les sites.

Sur le transect des sites de ramassage des fourrages, il a été observé l'ensablement des limites des champs par le sable entassé par les vents et un rabougrissement des arbustes fourragers liés au broutage continu par les animaux en vaine pâture. Un couvert végétal plus important a été observé sur les aires de pâturage et les jachères par rapport aux champs exploités; en outre, les résidus des plantes annuelles envahissantes (Sida cordifolia, Acanthospermum hispidum) et les pérennes non appétés récemment installées
(Pegularia tomentosa, Chrozophora brocchiana) ont été constatées.

Les enclaves pastorales sont caractérisées par une végétation menacée de coupe abusive dans tous les terroirs. Ces espaces étaient marqués par des souches des ligneux coupés, des signes de surpâturage étaient aussi visibles en guise d'une végétation ligneuse fourragère rabougrie à cause du broutage répétitif ainsi qu'une faible présence des résidus des herbacées fourragères. Il y avait sur ces lieux de pâturage des endroits compactés (pistes) dû au piétinement des animaux.

Tableau 1: Synthèse des réponses hiérarchisées de la perception des populations rurales sur les effets positifs du ramassage des fourrages sur l'environnement.

\begin{tabular}{ll}
\hline Effets positifs & Effectif \\
\hline Générer des revenus monétaires & 6 \\
\hline Améliorer les productions animales à travers l'alimentation & 4 \\
\hline Produire le fumier & 1 \\
\hline Lutter contre le feu de brousse & 1 \\
\hline Total & $\mathbf{1 2}$ \\
\hline
\end{tabular}

Tableau 2 : Principaux effets négatifs du ramassage des fourrages et leur effectif.

\begin{tabular}{ll}
\hline Effets négatifs & Effectif \\
\hline Dégradation des terres & 4 \\
\hline Perte de la biodiversité végétale et de végétation & 2 \\
\hline Rareté des ressources fourragères & 2 \\
\hline Baisse des productions animales & 1 \\
\hline Dégâts des animaux dans les champs & 1 \\
\hline Ensablement des vallées & 1 \\
\hline Apparition des espèces non appétées & 1 \\
\hline Total & $\mathbf{1 2}$ \\
\hline
\end{tabular}


Tableau 3 : Liste des espèces végétales menacées, disparues et en augmentation dans la zone de l'étude.

\begin{tabular}{|c|c|c|c|c|}
\hline Région & Village & Situation des espèces vég & les dans les terroirs & \\
\hline \multirow{8}{*}{ Maradi } & & Espèces menacées & $\begin{array}{l}\text { Espèces disparues } \\
\text { /terroir }\end{array}$ & $\begin{array}{ll}\text { Espèces } & \text { apparues } \\
\text { les 10 } & \text { dernières } \\
\text { années } & \end{array}$ \\
\hline & Djirataoua & $\begin{array}{l}\text { Alysicarpus ovalifolius } \\
\text { Commelina foreskalaei } \\
\text { Bauhinia rufescens }\end{array}$ & Zornia glochidiata & $\begin{array}{l}\text { Sida cordifolia } \\
\text { Acanthospermum } \\
\text { hispidum }\end{array}$ \\
\hline & El Kolta & $\begin{array}{l}\text { Alysicarpus ovalifolius } \\
\text { Schizachyrium exile } \\
\text { Andropogon gayanus } \\
\text { Piliostigma reticulatum } \\
\text { Bauhinia rufescens }\end{array}$ & $\begin{array}{l}\text { Dactyloctenium } \\
\text { aegyptium }\end{array}$ & $\begin{array}{l}\text { Sida cordifolia } \\
\text { Chrozophora } \\
\text { brocchiana } \\
\text { Calatropis procera } \\
\text { Acanthospermum } \\
\text { hispidum } \\
\text { Sclerocarya birea }\end{array}$ \\
\hline & Gadambo & $\begin{array}{l}\text { Alysicarpus ovalifolius } \\
\text { Commelina forskalaei } \\
\text { Guiera senegalensis } \\
\text { Albiza chevalieri }\end{array}$ & $\begin{array}{l}\text { Zornia glochidiata } \\
\text { Anona senegalensis }\end{array}$ & $\begin{array}{l}\text { Sida cordifolia } \\
\text { Chrozophora } \\
\text { brocchiana } \\
\text { Sclerocaria birea }\end{array}$ \\
\hline & Dan Kari & $\begin{array}{l}\text { Pennisetum pedicellatum } \\
\text { Piliostigma reticulatum } \\
\text { Guiera senegalensis }\end{array}$ & $\begin{array}{l}\text { Ctenium elegans } \\
\text { Dactyloctenium } \\
\text { aegyptium }\end{array}$ & Sida cordifolia \\
\hline & $\begin{array}{l}\text { Guidan } \\
\text { Matché } \\
\text { Daya }\end{array}$ & $\begin{array}{l}\text { Eragrostis tremula } \\
\text { Piliostigma reticulatum } \\
\text { Guiera senegalensis }\end{array}$ & $\begin{array}{l}\text { Dactyloctenium } \\
\text { aegyptium } \\
\text { Zornia glochidiata }\end{array}$ & $\begin{array}{l}\text { Sida cordifolia } \\
\text { Chrozophora } \\
\text { brocchiana } \\
\text { Calotropis procera } \\
\text { Pupalia lappocea }\end{array}$ \\
\hline & $\begin{array}{l}\text { Dadin } \\
\text { Kowa }\end{array}$ & $\begin{array}{l}\text { Cenchrus biflorus } \\
\text { Schizachyrium exile }\end{array}$ & $\begin{array}{l}\text { Dactyloctenium } \\
\text { aegyptium }\end{array}$ & Sida cordifolia \\
\hline & $\begin{array}{l}\text { Mondolo } \\
\text { Wali } \\
\text { Koira }\end{array}$ & $\begin{array}{l}\text { Zornia glochidiata } \\
\text { Diheteropogon hagerupii } \\
\text { Commelina foreskalaei } \\
\text { Andropogon gayanus } \\
\text { Combretum glutinosum } \\
\text { Aristida longiflora }\end{array}$ & $\begin{array}{l}\text { Detarium } \\
\text { microcarpum } \\
\text { Commiphora } \\
\text { africana }\end{array}$ & $\begin{array}{l}\text { Sida cordifolia } \\
\text { Pergularia } \\
\text { tomentosa }\end{array}$ \\
\hline Tillabéry & Warou & $\begin{array}{l}\text { Pennisetum pedicelLatum } \\
\text { Commilina forskalae } \\
\text { Eragrostis tremula } \\
\text { Leptadenia hastatae } \\
\text { Ipomoea dichroa } \\
\text { Alysicarpus ovalifolius } \\
\text { Aristida longiflora }\end{array}$ & $\begin{array}{l}\text { Schizachyrium exile } \\
\text { Annona } \\
\text { senegalensis } \\
\text { Detarium } \\
\text { microcarpum }\end{array}$ & $\begin{array}{l}\text { Sida cordifolia } \\
\text { Chrozophora } \\
\text { brocchiana }\end{array}$ \\
\hline
\end{tabular}




\begin{tabular}{|c|c|c|c|}
\hline Gatewane & $\begin{array}{l}\text { Pennisetum pedicelatum } \\
\text { Schoenefeldia gracilis } \\
\text { Leptadenia hastata } \\
\text { Eragrostis tremula } \\
\text { Ipomoea dichroa } \\
\text { Alysicarpus ovaliupolius } \\
\text { Aristida longiflora }\end{array}$ & $\begin{array}{l}\text { Schizachyrium exile } \\
\text { Annona } \\
\text { senegalensis }\end{array}$ & $\begin{array}{l}\text { Sida cordifolia } \\
\text { Chrozophora } \\
\text { brocchiana } \\
\text { Annona senegalensis }\end{array}$ \\
\hline Gogueze & $\begin{array}{l}\text { Zornia glochidiata } \\
\text { Diheteropogon hagerupii } \\
\text { Andropogon gayanus }\end{array}$ & $\begin{array}{l}\text { Pennisetum } \\
\text { pedicellatum }\end{array}$ & Sida cordifolia \\
\hline $\begin{array}{l}\text { Garbaye } \\
\text { Tombo }\end{array}$ & $\begin{array}{l}\text { Zornia glochidiata } \\
\text { Andropogon gayanus }\end{array}$ & $\begin{array}{l}\text { Pennisetum } \\
\text { pedicellatum }\end{array}$ & $\begin{array}{l}\text { Sida cordifolia } \\
\text { Calotropis procera }\end{array}$ \\
\hline $\begin{array}{l}\text { Karey } \\
\text { Gorou }\end{array}$ & $\begin{array}{l}\text { Schoenefeldia gracilis } \\
\text { Andropogon gayanus } \\
\text { Eragrostis tremula } \\
\text { Alysicarpus ovalifolius }\end{array}$ & Ipomoea dichroa & $\begin{array}{l}\text { Sida cordifolia } \\
\text { Acanthospermum } \\
\text { hispidum }\end{array}$ \\
\hline
\end{tabular}

Tableau 4 : Effets négatifs conjoints du ramassage des fourrages et du changement climatique sur l'environnement et les activités agro-sylvo-pastorales.

\begin{tabular}{ll}
\hline Effets négatifs & Fréquence $\mathbf{( \% )}$ \\
\hline Insuffisance des ressources pastorales & 29 \\
\hline Transhumance transfrontalière & 16 \\
\hline Faible productivité agricole et pastorale & 13 \\
\hline Perte de la biodiversité végétale & 11 \\
\hline Dégradation des terres & 11 \\
\hline Faible fertilité des sols & 10 \\
\hline Insécurité alimentaire pour les hommes et animaux & 10 \\
\hline Total & $\mathbf{1 0 0}$ \\
\hline
\end{tabular}

Tableau 5: Densité à l'hectare de ravins et des unités de surface dénudées parsite et par zone.

\begin{tabular}{llll}
\hline \multirow{2}{*}{ Site } & Zone & Variables & Nombre de ravins/ ha \\
\cline { 2 - 4 } & & $\begin{array}{l}\text { Nombre unités de surface dénudées } \\
\text { /ha }\end{array}$ \\
\hline Aires de pâturage & Maradi & $6,75^{\mathrm{a}}$ & $7,83^{\mathrm{b}}$ \\
\cline { 2 - 4 } & Tillabéry & $6,92^{\mathrm{a}}$ & $11,67^{\mathrm{b}}$ \\
\hline Champs & Maradi & $5,25^{\mathrm{a}}$ & $4,83^{\mathrm{a}}$ \\
\cline { 2 - 4 } & Tillabéry & $6,75^{\mathrm{a}}$ & $7,33^{\mathrm{b}}$ \\
\hline Jachères & Maradi & $6,08^{\mathrm{a}}$ & $6,33^{\mathrm{a}}$ \\
\cline { 2 - 4 } & Tillabéry & $7,33^{\mathrm{a}}$ & $8,08^{\mathrm{b}}$ \\
\hline Erreur standard & & 0,562 & 0,678 \\
\hline Probabilité & 0,264 & 0,004 \\
\hline $\begin{array}{l}\text { Dans une même colonne, pour une même unité agro-écologique les moyennes suivies d'une même lettre en ne sont pas } \\
\text { significativement différentes avec le test de Least Signicatif Difference au seuil de 5\%. }\end{array}$
\end{tabular}


Tableau 6: Moyennes de la matière organique végétale de surface et de la densité des ligneux dans les sites de ramassage des fourrages.

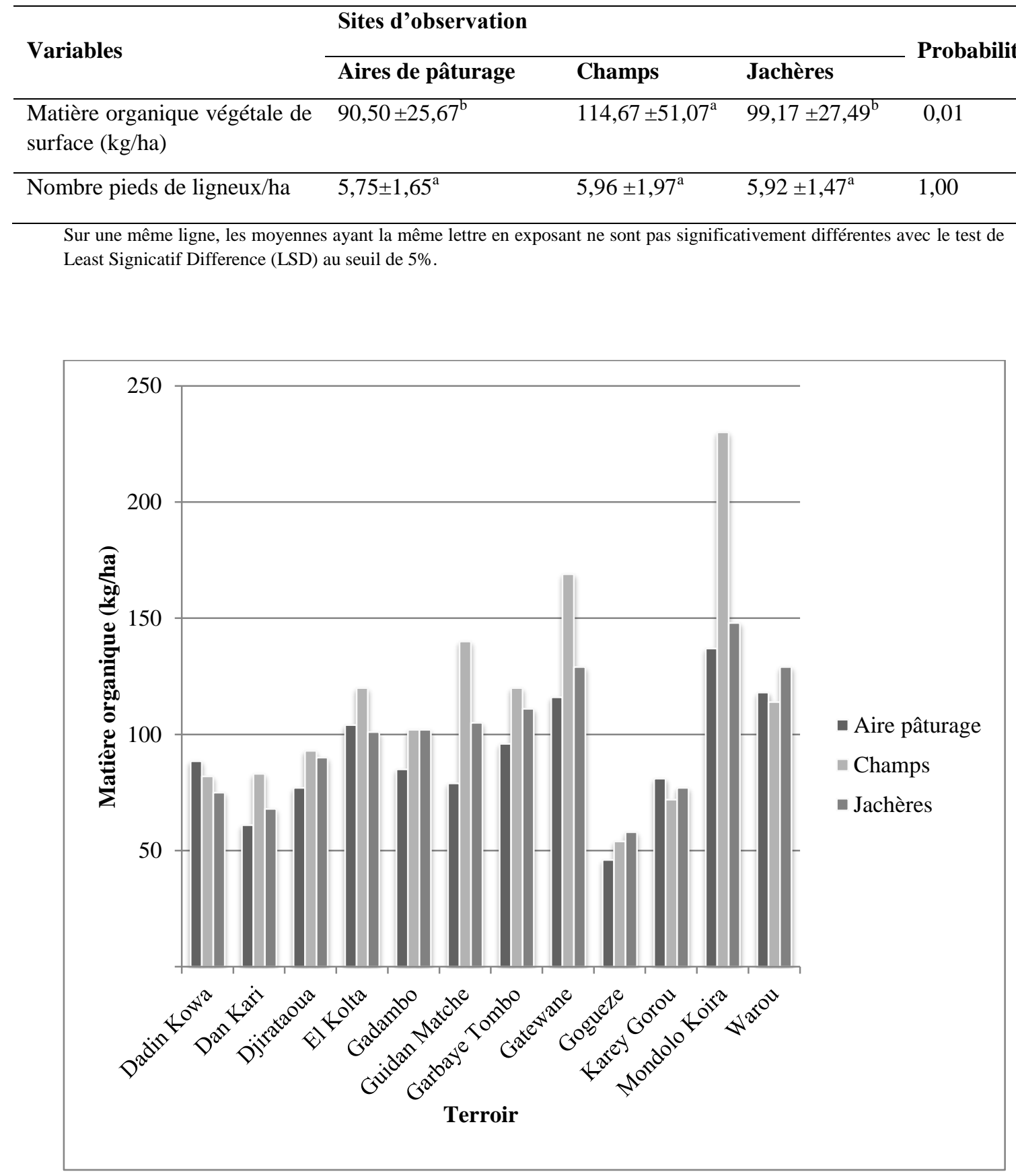

Figure 2 : Quantité de la matière organique végétale de surface par site et par terroir. 


\section{DISCUSSION}

Dans tous les villages objets de cette étude, les habitants interviewés sont conscients que les ressources naturelles sont le patrimoine commun et constituent la base de toutes les activités productives humaines. L'homme utilise les ressources naturelles à des fins de production: sols et terres pour l'agriculture, les pâturages pour l'élevage, pour la foresterie, pour la sylviculture et les cours d'eau et les lacs pour la pêche et l'abreuvement du cheptel. Le ramassage systématique des résidus de cultures et de fourrages naturels est un facteur de dégradation des ressources naturelles (sols, points d'eau, pâturages, espaces protégés) à partir desquelles les populations tirent leurs nourritures et leur revenu.

Les effets positifs de la collecte des fourrages concouraient à l'amélioration des conditions de vie des populations humaines et animales; par contre les effets négatifs constituent une véritable menace pour le bienêtre des hommes. Les populations rurales des zones de l'étude, ont évalué les effets du ramassage des fourrages par rapport à leurs besoins qui sont: la production de nourriture, l'accès à l'eau, le bien-être, le revenu et un environnement sain. Cela est conforme à la définition de dégradation physique et biologique de terres qui les font perdre leurs propriétés de support des plantes, de réservoir d'eau, de régulateur de température, de réservoir d'élements nutritifs, d'usine biologique de transformation et d'épuration (Gueye, 2013).

La similitude de l'état de dégradation (densité de ravins $\mathrm{ha}^{-1}$ ) des sites de prélèvement des fourrages entre les zones (Maradi et Tillabéry) peut s'expliquer par la forte pression exercée sur les ressources naturelles par les populations vivant dans la même zone agro-écologique, ayant les fortes densités humaines du pays $(32,4$ habitants $/ \mathrm{km}^{2}$ pour Tillabéry; 95,4 habitants $/ \mathrm{km}^{2}$ pour Mardi et 4567,4 habitants $/ \mathrm{km}^{2}$ pour Niamey) et les mêmes caractéristiques socio-économiques (agriculture, élevage, commerce, artisanat) (INS, 2016).
Ceci est en contradiction avec la conclusion faite par Larwanou et al. (2006) et Baggnian et al. (2012) selon laquelle le phénomène de reverdissement est surtout important dans les zones ayant une forte densité de population.

La faible densité de surfaces dénudées dans les champs à Maradi par rapport à celle de la zone de Tillabéry peut s'expliquer par l'usage très développé de matériels agricoles tels que la houe et la charrue dans la préparation des sols à Maradi, qui contribue à lutter contre cet état de dégradation des terres agricoles.

La forte pression animale exercée dans les enclaves pastorales et les jachères en toute saison, explique la densité élevée des surfaces dénudées dans ces espaces par rapport aux champs. Cela est un signe de dégradation des sols due au surpâturage en zones agricole et agro-pastorale où séjourne la majorité du cheptel national en saison sèche et où sont prélevés les fourrages distribués aux animaux au piquet en saison des pluies (Dan Gomma et al., 2017).

L'ensablement des limites des champs observé dans toutes les localités est une des conséquences du ramassage systématique des résidus de cultures, qui a favorisé les érosions hydrique et éolienne au niveau des terres agricoles. Les limites des champs sont en général arborées ou occupées par les adventices qui constituent des freins au sable et particules organiques transportés par le vent et l'eau de ruissellement (FAO, 2010b).

De façon générale, les moyennes de la quantité de la matière organique de surface par hectare très faibles $\left(<0,2\right.$ tonne $\left.\mathrm{ha}^{-1}\right)$ traduisent une exploitation minière de différents sites de prélèvement des fourrages. Elles sont nettement inférieures à la moyenne nationale de la matière organique $\mathrm{ha}^{-1} \mathrm{~d}$ 'un champ de mil à la récolte variant de 1,2 à 1,8 tonne ha ${ }^{-1}$ de 2011 à 2015 (INS, 2016) et aux 3,182 tonnes obtenus dans le champ de mil (Charles et al., 2010). Ce grand écart entre la quantité de la matière organique de surface à la récolte par hectare $\left(1200 \mathrm{~kg} \mathrm{ha}^{-1}\right)$ et celle évaluée en saison sèche (115 kg ha $\left.{ }^{-1}\right)$ démontre clairement le ramassage 
systématique des résidus de culture dans les régions de Maradi et de Tillabéry. La supériorité de la moyenne de la matière organique $\left(115 \mathrm{~kg} \mathrm{ha}^{-1}\right)$ dans les champs cultivés par rapport aux autres sites de prélèvement des fourrages s'explique par la présence du reliquat des tiges (partie inférieure dure) dans ces lieux, que les animaux ne parviennent pas à consommer en vaine pâture. L'abandon des tiges dans les champs après les récoltes à Mondolo et l'éloignement de cette localité par rapport au marché de Niamey expliquent les valeurs élevées de la matière organique obtenues dans ses champs et ses jachères. Ces résultats sont inférieurs à la moyenne nationale de $1700 \mathrm{~kg}$ $\mathrm{ha}^{-1}$ de tige de mil avec un coefficient de résidus de mil de 0,49 selon la DDP (2017), aux $126 \mathrm{~kg} \mathrm{ha}^{-1}$ obtenus dans les champs de mil à Say (Chaibou, 2011), aux $1399 \mathrm{~kg} \mathrm{ha}^{-1}$ obtenus dans les champs au bord de Goulbi Kaba par Illo Souley et al. (2018) et aux 3293 $\mathrm{kg} \mathrm{ha}^{-1}$ obtenus à la station de l'ICRISATSadoré par Pasternak et al. (2012). La moyenne de biomasse de surface végétale dans les jachères est inférieure aux $600 \mathrm{~kg} \mathrm{ha}^{-1}$ enregistrés dans la région de Tillabéry (DDP, 2016) et aux $2000 \mathrm{~kg} \mathrm{ha}^{-1}$ obtenus par Achard et al. (2001).

La faible densité des ligneux qui est similaire sur tous les sites s'explique par la forte pression humaine et animale que subit cette végétation en zones agricole et agropastorale. Le manque de végétation favorise l'érosion hydrique et éolienne qui réduit le potentiel productif des sols en les lessivant et en modifiant leurs propriétés physiques et biologiques. Cette densité des ligneux est nettement inférieure aux 26 pieds ha ${ }^{-1}$ trouvés dans le parc agro forestier de Dan SagaAguié dans la région de Maradi par Morou et al. (2016), aux 42,4 arbres ha ${ }^{-1}$ obtenus dans les champs par Issoufou et al. (2019), aux 9 pieds $\mathrm{ha}^{-1}$ sur les jachères rapportés par Samaké et al. (2011) et aussi inférieure aux 30 à 40 pieds ha $^{-1}$ recommandés en Régénération Naturelle Assistée dans les champs par Cunningham1 et Abasse (2005). En plus, l'état rabougri observé des ligneux fourragers dans les sites montre que cette végétation est menacée par la surexploitation par les animaux et les hommes qui les utilisent comme bois de chauffe, de service et d'œuvre dans toutes les zones. Les résultats montrent que la dégradation des terres résultante du ramassage de fourrages, est due à la mauvaise pratique d'exploitation et de gestion durable des terres et sont en harmonie avec ceux rapportés par Kohio et al. (2017).

\section{Conclusion}

La plupart des espèces fourragères naturelles sont menacées de disparition du fait de leur surexploitation, leur récolte avant la maturité et l'exportation de leurs semences. Cette étude a mis en exergue l'exploitation minière des terres, qui en les dépouillant de leur litière les empêche de remplir leurs fonctions de support pour les plantes, de réservoir d'eau et des éléments nutritifs. $\mathrm{La}$ collecte des fourrages dans les différents sites, bien qu'elle génère de revenu à ses acteurs, prive les sols de leur matière organique qui les enrichit et les protège contre la dégradation due aux facteurs climatiques et anthropiques. Ce travail a montré que la dégradation des terres et la perte de la biodiversité végétale sont plus accentuées en zone périurbaine de Niamey qu'à Maradi. La dégradation des terres réduit la productivité agricole, pastorale et agro forestière, exposant ainsi la population et le cheptel à l'insécurité alimentaire. La surexploitation des ressources fourragères dans les terroirs villageois, accentue la désertification, la perte de la biodiversité végétale et le déficit fourrager chronique qui réduit la productivité des animaux.

\section{CONFLIT D'INTERETS}

Les auteurs du présent manuscrit déclarent qu'il n'y a aucun conflit d'intérêts entre eux.

\section{CONTRIBUTIONS DES AUTEURS}

Dans la réalisation de la présente étude, ADG, SI et MB ont élaboré le protocole de recherche, collecté et analysé les données et rédigé le manuscrit. TA et ES ont participé à la relecture du document. 


\section{REMERCIEMENTS}

Les auteurs voudraient remercier les agriculteurs, agro-éleveurs et éleveurs des zones péri-urbaines de Maradi et de Niamey qui ont acceptés participer à cette étude.

\section{REFERENCES}

Abdou DG, Issa C, Maxime B, Eva S. 2017. Commercialisation et valeur nutritive des fourrages dans les centres urbains au Niger: cas des villes de Maradi et de Niamey. International Journal of Innovation and Applied Studies, 21: 508521. http://www.ijias.issr-journals.org/

Abdou DG, Molid. M'B, Ayssuwede S., Salissou I, Mahamadou MA, Siddo S, Ibro GD, Baye I, Mamann M, Chaibou I, Chaibou M, Moutari M, Chanono M. 2017. Evaluation technique et économique des formules alimentaires de blocs multi nutritionnels d'embouche ovine au Niger. Agronomie Africaine, 29(1): 1-11.

Soukaradji B, Abdou A, Lawali S, Aboubacar I, Mahamane A, Saadou M. 2017. Typologie des exploitations agricoles familiales : cas de la périphérie de la forêt protégée de Baban Rafi du Niger. Int. J. Biol. Chem. Sci., 11(3): 1096$1112 . \quad$ DOI: https://dx.doi.org/10.4314/ijbcs.v11i3.14

Baggnian I, Mohamadou A, Adamou MM, Lawali S, Adam T, Enfors E, Larwanou M, Tougiani A. 2012. Perceptions paysannes des tendances du reverdissement des zones dégradées au Niger. Université de Maradi. Journal des Sciences de l'Environnement, 1(1): 4352.

Chaibou I. 2011. Analyse des modes d'exploitation de la zone de transition de la Réserve Transfrontalière de Biosphère du $W$ au Niger. Thèse, UAM Niamey, Niger, $128 \mathrm{P}$.

Bielders LC, Dahiratou I, Maïmouna G. 2010. Contribution of arbuscular mycorrhizal fungi to pearl millet [Pennisetum glaucum (L.) R. Br.] nutrition on Sahelian acid sandy soils at various levels of soil degradation. Int. J. Biol. Chem. Sci., 4(4): 924-938. DOI: http://dx.doi.org/10.4314/ijbcs.v4i4.6297 4

Cunningham PJ, Abasse T. 2005. Reforesting the Sahel: Farmer Managed Natural Regeneration. In Domestication des espèces agro-forestières au Sahel: situation actuelle et perspectives, Kalinganire A, Niang A, Kone A. ICRAF Working Paper: ICRAF, Nairobi.

Dov Pasternak, Ali I, Ayantunde A. 2012. Determinants of the Availability of Adequate Millet Stover for Mulching in the Sahel. African Journal of Agriculture Research, 7(32): 526-535. DOI:10.589/AJAR11.1569.

Direction de Développement Pastoral (DDP)Ministère de l'Agriculture et de l'Elevage. 2016. Rapport de synthèse des résultats de la campagne pastorale 20162017. DDP-Ministère de l'Agriculture et de l'Elevage, $45 \mathrm{p}$.

Kohio EN, Toure AG, Sedogo MP, Ambouta KJ-M. 2017. Contraintes à l'adoption des bonnes pratiques de Gestion Durable des Terres dans les zones soudaniennes et soudano-sahéliennes du Burkina Faso. Int. J. Biol. Chem. Sci., 11(6): 29822989.

DOI: https://dx.doi.org/10.4314/ijbcs.v11i6.34

Edwige B, Comité inter-états de lutte contre la sécheresse au Sahel (CILSS). 2011. La mise à l'échelle de la gestion durable des terres au Sahel : des solutions pour une sécurité alimentaire durable. CILSS, $7 \mathrm{p}$.

Achard F, Hiernaux P, Banoin M. 2001. Les jachères fourragères naturelles et améliorées en Afrique de l'Ouest. La jachère en Afrique tropicale, Floret $\mathrm{C}$, Pontanier R, Serpentier G. John Libbey Eurotext : Paris@2001 ; 201-239.

Food and Agriculture Organization (FAO). 2010. Forêts 158. Lutte contre l'ensablement: l'exemple de la Mauritanie. FAO, 77 P.

Food and Agriculture Organization (FAO). 2010. Revue du secteur de l'élevage au Niger. FAO, 115 P. 
Food and Agriculture Organization (FAO), Centre International de Recherche pour le Développement (CIRAD). 2008. Système d'information sur le pastoralisme au Sahel. Atlas des évolutions des systèmes pastoraux au Sahel de 1970-2012.33. FAO, 33 p.

Hamissou MIS, Chaibou I, Soumana I, Laouali A, Mahamane A, Maxime B. 2018. Valeurs pastorales et productivités inter-décennales des parcours de la vallée de Goulbi Kaba au Niger. International Journal of Innovation and Applied Studies, 24(1): 220-239.

Baggnian I, Abdou L, Adam T, Mahamane A. 2019. Contribution de la migration aux renforcements des capacités socioéconomiques des populations de Tahoua dans la pratique de la régénération naturelle assistée (RNA): cas du village de Kolloma au Niger. Int. J. Biol. Chem. Sci., 13(1): 207-218. DOI: https://dx.doi.org/10.4314/ijbcs.v13i1.18

Gueye I. 2013. Application de la Télédétection aérospatiale pour l'évaluation de la dégradation des ressources naturelles: cas des sols de la région de Kaolack située dans le Bassin arachidier du Sénégal. Mémoire de Master en Chimie Biologie Aspects Analytiques, Université Cheikh Anta DIOP de Dakar, Faculté des Sciences et Techniques, $148 \mathrm{p}$.

Larwanou M, Abdoulaye M, Reij C. 2006. Etude de la régénération naturelle assistée dans la région de Zinder (Niger): Une première exploration d'un phénomène spectaculaire. International Resources Group: Washington DC, États Unis.
Ministère de l'Elevage du Niger. 2012. Stratégie de Développement Durable de l'Elevage (SDDEL) 2012-2035. Ministère de l'Elevage du Niger, $70 \mathrm{P}$.

Morou B, Ounani H, Amadou OA, Diouf A, Guero C, Mahamane A. 2016. Caractérisation de la structure démographique des ligneux dans les parcs agro forestiers du terroir de Dan Saga (Aguié, Niger). Int. J. Biol. Chem. Sci., 10(3): 1295-1311. DOI: 10.4314/ijbcs.v10i3.31

Ouodiouma S, Dakoua JM, Kalinganire A, Bayala J, Koné B. 2011. Régénération naturelle assistée (RNA). Gestion des arbres champêtres au Sahel. Manuel Technique No 16. World Agroforestry Centre (ICRAF), Programme Régional de l'Afrique de l'Ouest et du Centre, Nord Sahel, Bamako, Mali, 32 p.

Programme Régional d'Appui au Pastoralisme au Sahel (PRASP). 2015: L'Elevage pastoral au Sahel et en Afrique de l'Ouest.5 idées reçues à l'épreuve des faits. Inter-réseau de Développement rural. PRASP, $18 \mathrm{p}$.

Gonzaleza P, Tuckerb CJ, Sy H. 2012. Tree density and species decline in the African Sahel attributable to climate. Journal of Arid Environment, 78(2012): 55-64.

DOI:10.1016/j.jaridenv.2011.11.001

Illo Souley MH, Saley K, Chaibou I, Moussa BM, Mahamane A, Saadou M. 2018. Diversité Inter Décennale De La Végétation De La Vallée De Goulbi N'Kaba. European Scientific Journal, 14(9): $161-183 . \quad$ DOI: 10.19044/esj.2018.v14n9p161 